\title{
Desarrollo y Estudio Psicométrico de una Escala para Evaluar Conducta Prosocial en Adolescentes
}

\author{
Development and Psychometric Study of a Scale to Evaluate Prosocial Behavior in \\ Adolescents
}

\author{
Cinthia Balabanian ${ }^{1}$ y Viviana Lemos ${ }^{2}$
}

\begin{abstract}
Resumen
Los comportamientos prosociales, acciones intencionales que se llevan a cabo con el propósito de beneficiar a otros, implican consecuencias positivas para la sociedad, favoreciendo ampliamente las relaciones interpersonales. Dada la importancia de su evaluación y promoción, el objetivo de este trabajo fue la construcción y validación de un instrumento para evaluar la conducta prosocial en adolescentes de 12 a 18 años. Una versión conformada por 30 ítems fue administrada a una muestra de 492sujetos. Se realizó un Análisis Factorial Exploratorio, el cual arrojó evidencias de que la escala posee una estructura unidimensional. Se estudió la consistencia interna del instrumento, el poder discriminativo de los ítems y se realizaron análisis de validez de constructo hipotético, convergente y discriminante. A partir de los resultados obtenidosse concluye que el funcionamiento psicométrico del instrumento es adecuado, permitiendo evaluar de manera válida y confiable el constructo conducta prosocial en los adolescentes escolarizados argentinos.
\end{abstract}

Palabras clave: prosocialidad, evaluación psicológica, adolescencia

\begin{abstract}
Prosocial behaviors, intentional actions that are carried out with the purpose of benefiting others, imply positive consequences for society, favoring interpersonal relationships widely. Given the importance of its evaluation and promotion, the objective of this work was the construction and validation of an instrument to evaluate prosocial behavior in adolescents aged 12 to 18 years. A 30 -item version was administered to a sample of 492 subjects. An Exploratory Factorial Analysis was performed, which showed evidence that the scale has a one-dimensional structure. The internal consistency of the instrument, the discriminative power of the items, and validity analysis of hypothetical, convergent and discriminant constructs were studied. Based on the results obtained, it is concluded that the psychometric functioning of the instrument is adequate, allowing a valid and reliable evaluation of the construct of prosocial behavior in Argentinian students.
\end{abstract}

Keywords: prosociality, psychologicalevaluation, adolescence

\footnotetext{
${ }^{1}$ Licenciada en Psicología. Docente en Universidad Adventista del Plata. Becaria doctoral en Centro Interdisciplinario de Investigaciones en Psicología, Matemática y Experimental (CIIPME). Consejo Nacional de Investigaciones Científicas y Técnicas (CONICET). Alberdi 386. Libertador San Martín, Entre Ríos, Argentina. Tel.: 343-5055159. Correo: cinthiabalabanian@ gmail.com ${ }^{2}$ Doctora en Psicología. Docente titular en Universidad Adventista del Plata. Investigadora Independiente en Centro Interdisciplinario de Investigaciones en Psicología, Matemática y Experimental (CIIPME). Consejo Nacional de Investigaciones Científicas y Técnicas (CONICET). Castelli 180. Libertador San Martín, Entre Ríos, Argentina. Tel.: 343-4157611. Correo: viviananoemilemos@gmail.com
} 


\section{Introducción}

Durante los últimos años, la psicología positiva ha ido expandiendo su estudio sobre la conducta, con el objetivo de mejorar la calidad de vida de las personas y aumentar su felicidad. Desde esta perspectiva, el énfasis principal está puesto en fortalecer los recursos positivos de los individuos, incentivando en ellos las acciones que sean beneficiosas para su salud (Seligman \& Csikszentmihalyi, 2000).

En el marco de la psicología positiva, los comportamientos prosociales han sido definidos como acciones intencionales que se llevan a cabo con el propósito de beneficiar a otros (Inglés, Benavides, Redondo, García-Fernández, RuizEsteban, Estévez, \& Huescar, 2009; Roche Olivar, 1995; Roche Olivar, 1998).

Al momento de considerar las distintas formas en la que pueden manifestarse las conductas prosociales, se incluyen los actos de cooperación, condolencia, revalorización positiva del otro, ayuda verbal o física, consuelo frente a una dificultad o momento de angustia y rescate (Eisenberg, Fabes, \& Spinrad, 2006; Lemos \& Richaud de Minzi, 2013; Lemos \& Richaud de Minzi, 2014; Mestre Escrivá, Samper, Tur, Cortés, \& Nácher, 2006; Roche Olivar, 1995). Algunos ejemplos de este tipo de acciones seríanel voluntariado, ayudar y dar (Grant \& Dutton, 2012; Samper García, 2014).

De acuerdo con Roche Olivar (1998) la prosocialidad es un potente factor que conlleva consecuencias positivas en la sociedad y mejora la relación entre sus componentes. Asimismo, las conductas prosociales favorecerían ampliamente las relaciones interpersonales ya que aumentan la probabilidad de generar una reciprocidad positiva, solidaria y un trato cálido (Redondo Pacheco, Rueda Rueda, \& Amado Vega, 2013; Roche Olivar, 1995; Roche Olivar, 1997). Además, Grant y Dutton (2012) han mencionado que los comportamientos emprendidos con el objetivo de beneficiar a otras personas juegan un papel fundamental en la promoción de la educación, la protección de la salud, la disponibilidad para el alivio en situaciones de desastres y la lucha contra el hambre y la pobreza.

Cabe mencionar que además de las consecuencias positivas que se efectúan sobre quién es receptor de la ayuda, el comportamiento prosocial conlleva beneficios en los adolescentes y los jóvenes que llevan a cabo la conducta. Se han observado una serie de resultados positivos que aparecen en los adolescentes que realizan acciones prosociales, como por ejemplo: manifiestan mejores relaciones con sus compañeros, presentan mayores niveles de autoestima, sentido de pertenencia a la comunidad, mayor compromiso como ciudadano durante la transición de la adolescencia a la adultez y mejor rendimiento académico, así como en general una mejor adaptación en el curso de la vida (Bandura, Barbaranelli, Caprara, \& Pastorelli, 1996).

Así mismo, Grant y Dutton (2012) destacan que la conducta de dar puede promover una mayor felicidad que recibir. En este sentido, también revelan que la reflexión sobre el dar, en lugar de recibir, puede conducir a una mayor disposición y actitud servicial. De esta manera, el rol del benefactor adquiere importancia como objeto de estudio para la exploración de los mecanismos psicológicos implicados en esta experiencia, por ejemplo: responsabilidad, identidad como colaborador valioso, afirmación, realización y autoeficacia.

El estudio de la conducta prosocial (CP) ha sido enfocado tanto desde variables cognitivas (razonamiento) como a partir de variables emocionales. En este sentido, cabe mencionar que se destaca el papel de la empatía como motivadora de las acciones prosociales. Estudiada desde un enfoque multidimensional, ésta incluye aspectos cognitivos, como la toma de perspectiva y aspectos emocionales, como la capacidad para compartir sentimientos y la preocupación por el otro (Mestre Escrivá et al., 2006). La empatía, habilidad esencial para las personas, es considerada como precursora primordial de los comportamientos prosociales. La naturaleza intrínsecamente social de las personas, hace que la comprensión y el reconocimiento de los estados mentales de los demás sea una de las aptitudes necesarias para los seres humanos (López, Arán, \& Richaud de Minzi, 2014). Esta habilidad es aprendida en experiencias tempranas, transmitida principalmente por los padres a través de los estilos de crianza (Richaud de Minzi, 2014). Desde otra perspectiva, Caprara, Steca, Zelli y 
Capanna (2005) menciona que la empatía es un componente integral de la prosocialidad. En este sentido, existe una discusión teórica respecto a considerar a la empatía como una variable predictora y moderadora de las conductas de ayuda, o como parte de la misma tendencia prosocial (Auné, Abal, \& Attorresi, 2015a).

Entre los efectos positivos que se han observado en los adolescentes que se comprometen en actividades prosociales, se encuentra concretamente la disminución de comportamientos negativos como la agresión (Bandura et al., 1996). Este tipo de comportamientos se encuentran en contraposición con el comportamiento prosocial. La agresividad, definida como una conducta emocionalmente desadaptada, suele aparecer entre los adolescentes como consecuencia de escasos recursos positivos y acciones sociales activas (Del Barrio, Moreno Rosset, \& López Martínez, 2001). En este sentido, los patrones de desadaptación podrían disminuir a través del tiempo, si un repertorio conductual positivo es presentado como alternativa (Caprara, Luengo Kanacri, Zuffiano, Gerbino, \&Pastorelli, 2015). Así también, la realización de actos prosociales actúa previniendo la agresividad y la violencia, y reduciéndola en caso de que esté presente (Redondo Pacheco et al., 2013; Roche Olivar, 1998). Tal es así que las conductas disruptivas podrían inhibirse, detenerse o neutralizarse a partir de las conductas prosociales que estarían actuando de forma preventiva (Bandura et al., 1996).

Durante la adolescencia, el contexto escolar adquiere una gran relevancia debido a que en esta etapa los adolescentes pasan gran parte del día en instituciones educativas, Por esta razón surge en este contexto un mayor interés por fomentar conductas de ayuda basadas en la asertividad (Martínez González, Inglés Saura, Piqueras Rodríguez, \& Oblitas Guadalupe, 2010). En este sentido, el ámbito escolar cumple una función importante como agente de socialización, constituyendo un entorno de aprendizaje óptimo, en el que los niños y adolescentes pueden ser educados en valores prosociales (Greenberg, Weissberg, O'Brien, Zins, Fredericks, Resnik, \& Elias, 2003). Teniendo en cuenta que el comportamiento prosocial es una característica relativamente maleable, puede ser fomentado adecuadamente a través de acciones educativas apropiadas (Carlo, Fabes, Laible, \& Kupanoff, 1999).

Los resultados positivos que se han observado en contextos escolares, pueden explicarse considerando que los adolescentes que se comportan prosocialmenteallí, son más propensos a establecer un entorno social de apoyo, en el que sentirse aceptados y apoyados por sus pares y profesores (Jennings \& Greenberg, 2009). En suma, existe una cantidad considerable de evidencia empírica que sugiere que el fomento de la conducta prosocial en el ámbito escolar contribuye al aprendizaje y la adaptación del estudiante, a la vez que actúa como protector de resultados negativos como la agresión, el comportamiento antisocial y el rechazo de los compañeros (Caprara et al., 2015).

Por todo esto, en el marco del vínculo escolar del adolescente se destaca la importanciadel desarrollo de programas con el objetivo de promover conductas prosociales, y evaluar la eficacia de tales intervenciones (Garaigordobil, 2005).

En cuanto a la evaluación de las conductas prosociales en adolescentes, si bien es posible encontrar algunos instrumentos que operacionalizan este constructo incluso en nuestro idioma, se ha observado quelos cuestionarios existentes, además de operacionalizar la conducta prosocial propiamente dicha, tienden a incluir ítems que evalúan otras variables relacionadas desde el punto de vista teórico. Un ejemplo de este caso es el Cuestionario de Conducta Prosocial (CCP) diseñado por Martorell, González, Ordoñez y Gómez (2011) para adolescentes españoles de entre 10 y 17 años, que contiene cuatro dimensiones que fueron denominados: Empatía, Respeto, Relaciones Sociales y Liderazgo. Se considera que estos componentes hacen referencia a otros aspectos, que si bien están asociados con la prosocialidad, implicarían conceptos diferentes que podrían ser evaluados de manera independiente. Por otro lado, las distintas tendencias prosociales adolescentes han sido operacionalizadas por Carlo y Randall (2002) a través del Prosocial TendenciesMeasure (PTM). Richaud de Minzi, Mesurado y Kohan Cortada (2012) realizaron una adaptación de dicho instrumento al español, encontrando las 
dimensiones de: altruista, pública, anónima y respuesta. Se ha observado que el PTM hace referencia a diferentes motivaciones que impulsarían acciones prosociales, y no tipos de comportamientos propiamente dichos.

Un instrumento que evalúa con una estructura multidimensional los distintos aspectos teóricos del comportamiento prosocial, es el desarrollado por Auné, Abal y Attorresi (2015b), el cual operacionaliza los factores: comportamiento empático, altruismo, ayuda y acciones de compartir. Sin embargo, este instrumento es dirigido a población adulta.

Dada las implicancias que tiene la prosocialidad en la prevención y promoción positivadel desarrollo humano y los beneficios que conlleva la realización de actos sociales positivos, es de gran relevancia diseñar yllevar a cabo programas de intervención que promuevan las conductas de ayuda. Dado que es fundamental que pueda evaluarse la eficacia y efectividad de tales intervenciones,es necesario contar con una medida válida y confiable del constructo. En este contexto, y considerando que no existe un instrumento adecuado para evaluar la conducta prosocial adolescente en nuestro medio, se propone como objetivo la construcción de una escala que permita operacionalizar la prosocialidad en adolescentes de 12 a 18 años de edad $\mathrm{y}$ el estudio de sus propiedades psicométricas.

\section{Método}

De acuerdo con la conceptualización de Montero y León (2007), el estudio llevado a cabo en este trabajo es de carácter instrumental. Este tipo de investigaciónbusca el desarrollo de pruebas, en las que se incluye el diseño o estudio de las propiedades psicométricas de las mismas. En este sentido, se pretendió construir la Escala de Conducta Prosocial Adolescentey evaluar su desempeño psicométrico.

A continuación, se explicitan los participantes,procedimientos seguidos, resultados y conclusiones obtenidas.

Procedimientos seguidos en la redacción de los ítems

Para llevar a cabo esta instancia, se escogió una institución privada confesional de nivel medio, donde se solicitó la autorización correspondiente. Se decidió trabajar con adolescentes de entre 12 y 18 años debido a que, en comparación con los sujetos de etapas anteriores, los adolescentes poseen una conciencia mayor de sus estados afectivos (López \& Ortiz, 2005).

Una vez obtenido el permiso por parte del colegio, se realizó el contacto con algunos cursos, y siguiendo las normas éticas correspondientes, se repartieron los consentimientos informado para que los sujetos que desearan participar los hicieran firmar por sus respectivos padres. Luego, de manera voluntaria, los adolescentes que fueron autorizados participaron de los talleres, que se realizaron por grupos de aproximadamente 30 sujetos, en un espacio brindado por la institución. Se comenzó la actividad presentado el concepto de conducta prosocial,algunos ejemplos de este tipo de comportamiento e imágenes que pudieran servir como disparadores.

Estos talleres fueron realizados con el objetivo de que los adolescentes pudieran identificar y mencionar ejemplos de comportamientos de ayuda que resultaran cotidianos para ellos, relevando las expresiones utilizadas por los mismos adolescentes. Para ello se utilizó la siguiente pregunta, reiteradas veces durante el taller: ¿Cuáles serían para ustedes ejemplos concretos de conductas prosociales?

Se llevaron a cabo además entrevistas conalgunos docentes que están en contacto diario con alumnos de nivel medio, presentándoseleslos objetivos del estudio y solicitándoles la misma consigna dada al grupo de alumnos: ¿Cuáles serían para ustedes ejemplos concretos de conductas prosociales adolescentes?

Estos informantes clave aportaron respuestas similares a las obtenidas en los talleres con los adolescentes. Luego se realizó la transcripción de las respuestas obtenidas en las entrevistas, con el propósito de preparar el material para el análisis. A partir de los ejemplos concretos de conductas prosociales mencionados por los adolescentes e informantes clave, se procedió al análisis de las respuestas obtenidas, mediante la codificación emergente de las mismas.

Posteriormente, se redactó un pool de 71 ítems que conformaron la escala preliminar. Se elaboró el esquema del instrumento, determinando las opciones de respuesta y el formato en general. Se eligió una escala tipo Likert de cinco puntos, 
expresados en grados de frecuencia ( $1=$ Nunca, $2=$ Alguna vez, 3=Muchas veces, $4=$ Casi siempre y 5=Siempre). Se agregó la opción "No aplica" para conocer cuáles ítems podrían resultarirrelevantes para los sujetos, es decir, con cuáles situaciones no se identificaban.

A continuación, se realizó un análisis de validez de contenido del instrumento, a través de la consulta a jueces expertos, quienes realizaron aportes específicos y sugerencias en cuanto a los términos utilizados, la pertinencia de los ítems y la representatividad en términos de tipos de conducta prosocial evaluadas. Aunque ningún ítem fue eliminado en esta instancia, se corrigió la redacción de algunos reactivos.

Procedimientos seguidos en el estudio del instrumento

Con el objetivo de estudiar las propiedades psicométricas de la versión preliminar, la escala de 71 ítems fue administrada en forma grupal a una muestra piloto de 142 adolescentes de ambos sexos, de entre 12 y 18 años,alumnos de un colegio privado de la ciudad de Libertador San Martín, Entre Ríos. El promedio de edad fue de 15.03 años $(D E=1.49)$.

A continuación, se estudióel poder discriminativo de los ítems, la consistencia interna y se realizaron análisis factorial exploratorio (AFE). Luego de sucesivos AFE utilizando el método de componentes principales y rotación Oblimin, en los que se fue depurando la matriz factorial, descartando aquellos ítems con baja comunalidad o bajo peso en el factor que se pretendía operacionalizar, se llegó a una estructura unidimensional clara y simple compuesta por 30 ítems. La consistencia interna resultó satisfactoria y todos los ítems discriminativos. Los resultados de esta versión preliminar se encuentran en Balabanian y Lemos (2016).

La versión resultante de 30 ítems fue administrada a una segunda muestra intencionalcompuesta por 492 adolescentes de ambos sexos, de entre 12 y 18 años, pertenecientes a colegios públicos y privados en la ciudad de Colón, Entre Ríos. El promedio de edad fue de 14.78 ( $D E=1.86)$. Los sujetos participaron de forma voluntaria y con la previa autorización de sus padres. Se aplicaron también otras escalas con el objetivo de obtener evidencias de validez de constructo, convergente y discriminante del instrumento desarrollado.

Los datos obtenidos fueron cargados en el programa estadístico SPSS, para su posterior análisis. En esta instancia, se conservaron todos los protocolos, ya que no hubo test con alto porcentaje de respuestas incompletas.

Para conocer las propiedades psicométricas de la escala, se realizaron los siguientes procedimientos estadísticos:

Con el objetivo de examinar el poder discriminativo de los ítems, se utilizó el criterio de grupos contrastantes, para analizar luego las respuestas mediante la prueba $t$ de diferencia de medias para muestras independientes. Esto se llevó a cabo para conocer si existían diferencias estadísticamente significativas entre el cuartil superior (sujetos que puntúan más alto, percentil por encima de 75) y el cuartil inferior (quienes puntuaron más bajo, percentil inferior a 25) en la variable que se pretende medir (Cohen \& Swerdlik, 2001).

Se estudió la pertinencia de efectuar un análisis factorial mediante la prueba de esfericidad de Bartlett y el índice de adecuación muestral Kaiser-Meyer-Olkin. A continuación, se realizó un nuevo Análisis Factorial Exploratorio (AFE) a través del método de componentes principales con rotación oblicua, partiendo del supuesto teórico de que los factores están correlacionados entre sí.

Se calculó el índice de alpha de Cronbach para la escala general, con el fin de evaluar la confiabilidad desde el aspecto de la consistencia interna.

Finalmente, para obtener evidencias de la validez de constructo convergente, discriminante y de constructo hipotético del instrumento, se realizaron análisis de correlaciones $r$ de Pearson. De esta manera, se estudió la relación entre la escala estudiada con otras puntuaciones que evalúan constructos semejantes y diferentes.

1. Para el estudio de la validez de constructo convergente, se eligió la Escala de Conducta Prosociales (Caprara et al., 2005; Del Barrio et al., 2001). Dicha escala ofrece una medida global de prosocialidad y está compuesta por 16 ítems. Se aplicó para estudiar si existe una correlación significativa y positiva con las puntuaciones del instrumento de conducta prosocial construido. Este instrumento ha sido utilizado en nuestro 
contexto, encontrándose propiedades psicométricas adecuadas (Lemos, 2009; Lemos, Hendrie, \& Oros, 2015; Richaud de Minzi, Lemos, \& Mesurado, 2011; Richaud de Minzi, 2015).

2. Para el estudio de la validez de constructo discriminante, se utilizó el Cuestionario de Agresión Física y Verbal, (Caprara et al., 2005; Del Barrio et al., 2001). Éste consta de 15 ítems a partir de los cuales es posible evaluar la conducta de dañar de forma física o verbal a otras personas.Se utilizó esta escala para conocer si existe correlación significativa y negativa con la escala de conductas prosociales. Al igual que la escala de Caprara y Pastorelli, de conducta prosocial, este instrumento también ha sido utilizado en nuestro contexto, encontrándose propiedades psicométricas adecuadas (Lemos, 2009; 2012; Richaud de Minzi, 2015).

3. Para el estudio de la validez de constructo hipotético se evaluó la empatía, mediante la aplicación de la adaptación argentina (Richaud de Minzi, 2008) delInterpersonal ReactivityIndex (IRI) de Davis (1980). Esta escala consta de cuatro dimensiones: preocupación empática, toma de perspectiva, fantasía y malestar emocional. En este trabajo se consideraron las dos primeras dimensiones mencionadas, con el objetivo de conocer si existe una correlación significativa y positiva con la conducta prosocial evaluada a través del instrumento construido, tal como sería esperable desde el punto de vista teórico (López et al., 2014).

\section{Resultados}

Luego de realizarse los análisis mencionados para conocer el poder discriminativo de los reactivos del instrumento, es posible mencionar que los 30 ítems que integran la versión final del instrumento discriminan entre los sujetos que obtuvieron puntuaciones menores en el atributo y quienes obtuvieron una mayor puntuación $(p<$ .001). Ver Tabla 1.

El índice de adecuación muestral KaiserMeyer-Olkin arrojó un valor de .889 mientras que la prueba de esfericidad de Bartlett un $X^{2}$ de Bartlett $=3848.198 \quad(p=.00)$. Dado que estos resultados fueron satisfactorios, se llevó a cabo un análisis factorial para conocer la estructura de la matriz, utilizando el método de rotación Oblimin. Considerando la pendiente de Cattel (ver Gráfico 1) y la varianza total explicada, se encontró que los 30 ítems pueden ser explicados claramente por una estructura unidimensional. La presencia de un factor subyacente explicaría el $26.84 \%$ de la varianza total. Todos los ítems excepto uno, están presentes en el factor con un peso mayor a .30. Ver Tabla 2.

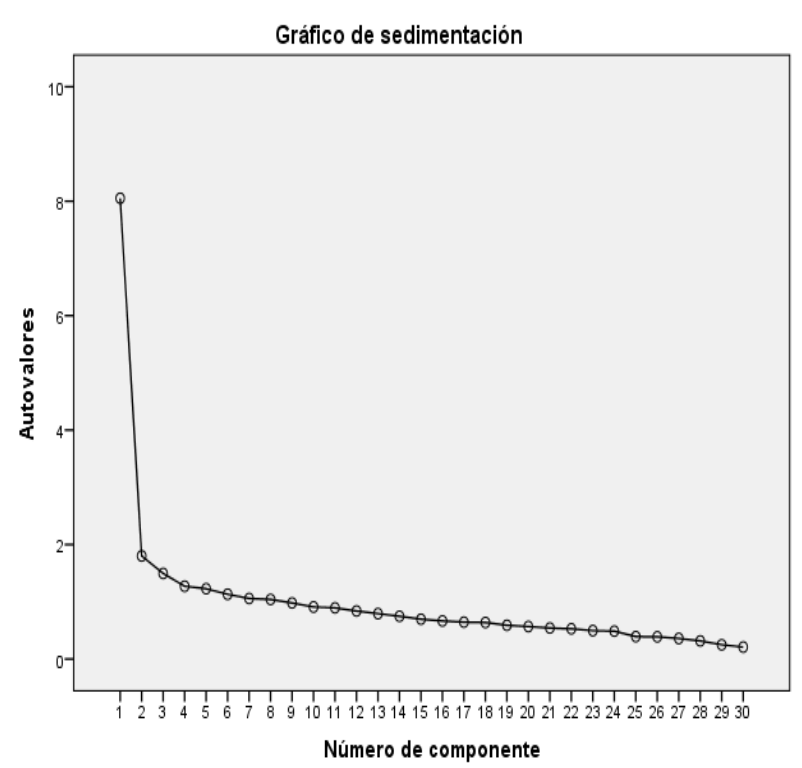

Gráficol. Pendiente de Cattel

En cuanto a la confiabilidad del instrumento, se evaluó la consistencia interna, obteniéndose un coeficiente Alpha de Cronbach igual a .90 para la escala global. Este valor coincide con el resultado obtenido en los adolescentes que conformaron la primera muestra piloto.

Con respecto al análisis de la validez de constructo convergente, los resultados obtenidos indican que la conducta prosocial, evaluada a través del cuestionario de Caprara et al. (2005) se correlaciona de forma positiva y significativa, desde el punto de vista estadístico $(r=.684 ; p=.01)$, con la escala construida para adolescentes.

Así también, la empatía operacionalizada a través de las dimensiones preocupación empática y toma de perspectiva del IRI (Davis, 1980; Richaud de Minzi, 2008) correlacionó de forma positiva y significativa con las puntuaciones del instrumento de conducta prosocial ( $r=.552$; $p=.01$ ), dando evidencias de validez de constructo hipotético.

Por último, tal como se esperaba teóricamente, la escala de agresión física y verbal 
Tabla 1. Poder discriminativo de los ítems de la versión final de la escala

\begin{tabular}{|c|c|c|c|c|c|c|}
\hline & \multicolumn{2}{|c|}{ Grupo bajo } & \multicolumn{2}{|c|}{ Grupo alto } & \multirow{2}{*}{$t$} & \multirow{2}{*}{$p$} \\
\hline & $M$ & $D E$ & $M$ & $D E$ & & \\
\hline Ítem 1 & 2.90 & 1.150 & 4.17 & .982 & -9.760 & .000 \\
\hline Ítem 2 & 2.79 & 1.182 & 3.88 & 1.192 & -7.548 & .000 \\
\hline Ítem 3 & 2.08 & .925 & 3.54 & 1.156 & -11.384 & .000 \\
\hline Ítem 4 & 2.99 & 1.110 & 3.90 & 1.084 & -6.828 & .000 \\
\hline Ítem 5 & 3.01 & 1.201 & 4.62 & .729 & -13.438 & .000 \\
\hline Ítem 6 & 1.50 & .829 & 2.86 & 1.310 & -10.080 & .000 \\
\hline Ítem 7 & 2.38 & 1.086 & 3.69 & 1.244 & -9.180 & .000 \\
\hline Ítem 8 & 2.11 & .961 & 3.56 & 1.264 & -10.567 & .000 \\
\hline Ítem 9 & 2.42 & 1.076 & 3.99 & 1.117 & -11.777 & .000 \\
\hline Ítem 10 & 1.68 & .972 & 2.75 & 1.353 & -7.457 & .000 \\
\hline Ítem 11 & 2.36 & 1.022 & 4.28 & .863 & -16.572 & .000 \\
\hline Ítem 12 & 2.04 & .977 & 4.11 & 1.013 & -17.009 & .000 \\
\hline Ítem 13 & 1.77 & .895 & 3.22 & 1.331 & -10.435 & .000 \\
\hline Ítem 14 & 2.52 & .943 & 4.42 & .879 & -17.004 & .000 \\
\hline Ítem 15 & 1.77 & .828 & 3.50 & 1.215 & -13.555 & .000 \\
\hline Ítem 16 & 2.37 & 1.124 & 4.27 & 1.077 & -14.157 & .000 \\
\hline Ítem 17 & 1.78 & .843 & 3.37 & 1.277 & -11.984 & .000 \\
\hline Ítem 18 & 2.29 & 1.105 & 4.05 & .983 & -13.815 & .000 \\
\hline Ítem 19 & 2.59 & 1.191 & 4.65 & .657 & -17.683 & .000 \\
\hline Ítem 20 & 1.96 & 1.191 & 3.14 & 1.477 & -7.192 & .000 \\
\hline Ítem 21 & 2.94 & 1.156 & 4.49 & .828 & -12.760 & .000 \\
\hline Ítem 22 & 3.47 & 1.358 & 4.75 & .671 & -9.941 & .000 \\
\hline Ítem 23 & 3.19 & 1.225 & 4.62 & .729 & -11.770 & .000 \\
\hline Ítem 24 & 2.33 & .958 & 4.20 & .830 & -17.048 & .000 \\
\hline Ítem 25 & 2.53 & 1.163 & 4.35 & .825 & -14.950 & .000 \\
\hline Ítem 26 & 2.55 & 1.023 & 4.04 & .952 & -12.302 & .000 \\
\hline Ítem 27 & 3.42 & 1.245 & 4.73 & .620 & -11.061 & .000 \\
\hline Ítem 28 & 2.37 & .792 & 4.05 & .926 & -16.021 & .000 \\
\hline Ítem 29 & 2.17 & .940 & 4.14 & 1.002 & -16.605 & .000 \\
\hline Ítem 30 & 2.03 & 1.096 & 3.58 & 1.305 & -10.497 & .000 \\
\hline
\end{tabular}

(Caprara et al., 2005; Del Barrio et al., 2001) correlacionó de forma negativa y significativa $(r=-$ .195; $p=.01)$ con la conducta prosocial evaluada mediante el instrumento construido en este estudio, proporcionando evidencia de validez de constructo discriminante. Ver Tabla 3.

\section{Conclusiones}

Tal como lo establece la psicometría, los indicadores de validez y confiabilidad evalúan la calidad de una prueba psicológica (MartínezArias, Hernández-Lloreda, \& Hernández-Lloreda,
2006). Por tanto, ambos aspectos fueron analizados en el presente estudio, considerando distintos tipos de validez, análisis del poder discriminativo de los ítems y consistencia interna del instrumento.

A partir de los resultados obtenidos, se observó que todos los ítems que componen la prueba construida tienen la capacidad de discriminar de forma significativa entre los sujetos que presentan menores y mayores valores en la puntuación de conductas prosociales evaluadas.

Con respecto a la confiabilidad del instrumento, analizada a partir de la consistencia 
Tabla 2. Matriz de configuración y peso de cada ítem en el factor

\begin{tabular}{|c|c|}
\hline & $\begin{array}{c}\text { Componente } \\
1\end{array}$ \\
\hline 1. Presto algo por un tiempo si alguien necesita algo que yo tengo. & .440 \\
\hline 2. Pongo la mesa a la hora del almuerzo/cena. & .220 \\
\hline 3. Ayudo a un compañero a estudiar cuando le cuesta un tema. & .502 \\
\hline 4. Mis compañeros me consideran una persona amigable. & .405 \\
\hline 5. Doy ánimo cuando un compañero está triste o cansado. & .614 \\
\hline 6. Si encuentro una persona mayor con bolsas del supermercado, la ayudo. & .446 \\
\hline 7. Hago regalos, aunque sean detalles. & .374 \\
\hline 8. En un trabajo práctico, incluyo a quienes no están en ningún grupo. & .446 \\
\hline 9. Explico un concepto a quién no haya entendido. & .528 \\
\hline 10. Ordeno el aula antes de retirarme. & .344 \\
\hline 11. Felicito a otro cuando tiene una buena idea o hace algo bien. & .583 \\
\hline 12. Me acerco a hablar con un compañero que es nuevo en la escuela. & .600 \\
\hline 13. Ayudo a el/la profesor/a cuando está muy cargado/a y no puede llevar sus cosas. & .466 \\
\hline 14. Ayudo a un compañero si se quedó en el dictado o tomando apuntes. & .596 \\
\hline 15. Participo en actividades solidarias. & .565 \\
\hline 16. Defiendo a un compañero cuando está siendo agredido. & .614 \\
\hline 17. Invito a mis compañeros a las actividades sociales, no sólo a mi grupo de amigos. & .562 \\
\hline 18. Ayudo a una persona si se tropieza o se cae. & .567 \\
\hline 19. Consuelo a un compañero que está llorando. & .705 \\
\hline 20. Saco la basura cuando es necesario, sin que me lo pidan. & .230 \\
\hline 21. Presto mis fotocopias si un compañero necesita. & .484 \\
\hline 22. Escucho atentamente los problemas de mis amigos cuando quieren desahogarse. & .557 \\
\hline 23. Escucho a personas que necesitan ser oídas. & .590 \\
\hline 24. Acompaño a un compañero si veo que está solo. & .623 \\
\hline 25. Felicito a un compañero cuando se saca una buena nota. & .527 \\
\hline 26. Aporto información cuando me realizan una consulta. & .491 \\
\hline 27. Intento hacer reír a alguien que está triste. & .503 \\
\hline 28. Apoyo la propuesta de un compañero y lo incentivo. & .592 \\
\hline 29. Interactúo con los compañeros nuevos. & .618 \\
\hline 30. Pido a mis compañeros que dejen de conversar cuando habla el profesor. & .376 \\
\hline
\end{tabular}

Tabla 3. Correlaciones para evidencias de validez de constructo hipotético, convergente y discriminante

\begin{tabular}{cccc}
\hline & $\begin{array}{c}\text { Conducta } \\
\text { prosocial } \\
\text { (Caprara) }\end{array}$ & $\begin{array}{c}\text { Agresión } \\
\text { física y } \\
\text { verbal }\end{array}$ & Empatía \\
\hline $\begin{array}{c}\text { Conducta } \\
\text { prosocial } \\
\text { (Balabanian \& } \\
\text { Lemos, 2016) }\end{array}$ & $.684 * *$ & $-.195 * *$ & $.552 * *$ \\
$* * p=.01$ & & & \\
\hline
\end{tabular}

interna de la misma, puede decirse que el coeficiente resultó adecuado para la prueba, incluyendo el conjunto de 30 ítems.

Con relación a la validez factorial de la escala, los resultados encontrados fueron satisfactorios. Se obtuvo una matriz de estructura simple y unifactorial. Debido a que no se encontraron sub dimensiones dentro de la escala total, es posible mencionar que todos los reactivos del instrumento estarían brindando información acerca de la prosocialidad evaluada de forma global.

El hecho de que se haya encontrado un solo factor subyacente al conjunto de ítems, permite inferir que, aunque el instrumento operacionalice algunos tipos específicos de comportamientos prosociales que son mencionados por la literatura existente, dichos comportamientos tendrían una alta correlación entre sí, lo que queda evidenciado en el elevado valor obtenido al analizar la consistencia interna del instrumento.

Se observó que los ítems 2 ("pongo la mesa a la hora del almuerzo") y 20 ("saco la basura cuando es necesario...") presentaron un peso inferior en el factor, en comparación con los demás reactivos. Una posible explicación sería que estos dos ítems hacen referencia a conductas que pueden ser consideradas buenos hábitos dentro del hogar. Así también, el ítem 10 (“ordeno 
el aula antes de retirarme"), que representa un peso un poco más elevado que los ítems mencionados, pero igualmente inferior al resto, podría referirse a un comportamiento de buenos modales dentro del ámbito escolar.

Muchos de los reactivos que conforman el instrumento de este estudio, se corresponderían con la categorización de Roche Olivar (1995, citado en Roche Olivar 1998) quien realizó una de las primeras clasificaciones de las conductas prosociales. Tal es el caso de los ítems 6 ("si encuentro una persona mayor con bolsas del supermercado, la ayudo"), 13 ("ayudo a el/la profesor/a cuando...") y 18 ("ayudo a una persona si se tropieza o cae") que podrían incluirse en la categoría de ayuda física mencionada por dicho autor. Por otro lado, los ítems 3 ("ayudo a un compañero a estudiar..."), 9 ("explico un concepto..."), 14 ("ayudo a un compañero si se quedó en el dictado...") y 26 (“aporto información...") podrían ser ejemplos de ayuda verbal, categoría también presentada por el autor mencionado. Otra de las categorías que se ve representada es el consuelo verbal, en los ítems 5 (“doy ánimo...”) y 19 (“consuelo a un compañero..."). Así también los ítems 11 ("felicito a otro..."), 25 ("felicito a un compañero cuando se saca una buena nota") y 28 ("apoyo la propuesta...") serían ejemplos de la categoría confirmación y valorización positiva del otro. Siguiendo con este autor, se hace referencia a la escucha profunda como parte de las conductas prosociales, y podría comprenderse a través de los ítems 22 ("escucho atentamente los problemas...") y 23 ("escucho a personas que necesitan..."). Por último, existen dos grupos de conductas que estarían representadas en algunos ítems: por lado la solidaridad que se vería en los ítems 1 ("presto algo..."), 15 ("participo en actividades solidarias") y 21 ("presto mis fotocopias...") y la presencia positiva y unidad, que estaría representada en los ítems 8 (“...incluyo a quienes no están en ningún grupo"), 12 ("me acerco a hablar..."), 17 ("invito a mis compañeros..."), 24 ("acompaño a un compañero...") y 29 ("interactúo con los compañeros nuevos").

Los ítems de este instrumento, incluyen las conceptualizaciones teóricas realizadas años atrás por Roche Olivar (1995, citado en Roche Olivar,
1998), como así ejemplos de comportamientos propios y de otros, dados por los propios adolescentes en la actualidad.

Finalmente, las correlaciones obtenidas entre el instrumento de comportamiento prosocial construido y las pruebas utilizadas para evaluar distintos tipos de validez, fueron consistentes con lo esperado teóricamente y significativas en todos los casos.Con respecto al análisis de validez convergente, tal como mencionan Tornimbeni, Pérez y Olaz (2008) la convergencia estaría dada por correlaciones relativamente altas entre las puntuaciones de instrumentos de medición diseñados para evaluar un constructo común. De este modo, los resultados indicaron una correlación positiva y significativa entre el CP y el instrumento en estudio. Respecto a la evaluación de la empatía, la correlación positiva y significativa que se obtuvo está de acuerdo con el papel de esta variable como motivadora y precursora del comportamiento prosocial (Mestre Escrivá et al., 2006; López et al., 2014).Por otro lado, las puntuaciones observadas en la evaluación de la agresión presentaron una correlación negativa con las acciones de ayuda, aportando evidencias de que ambas conductas son contrapuestas (Bandura et al., 1996; Caprara et at., 2015; Del Barrio et al., 2001; Redondo Pacheco et al., 2013; Roche Olivar, 1998).

Teniendo en consideración los análisis realizados,se destaca que la Escala de Conductas Prosociales en Adolescentes posee diferentes evidencias de validez y confiablidad, y, por lo tanto, podría utilizarse en este contexto para evaluar los comportamientos de ayudaen adolescentes escolarizados.

Futuras investigaciones podrían realizar nuevos estudios sobre la escala, para aportar evidencias adicionales de validez. Se proyecta además realizar un Análisis Factorial Confirmatorio, y una propuesta del modelo desde la Teoría de Respuesta al Ítem.

\section{Referencias}

Auné, S. E., Abal, F. J. P., \& Attorresi, H. F. (2015a). Antagonismos entre concepciones de empatía y su relación con la conducta prosocial. Revista de Psicología, 17(2), 137149. doi:10.18050/revpsi.v17n2a7.2015 
Auné, S. E., Abal, F. J. P., \& Attorresi, H. F. (2015b). Diseño y construcción de una escala de conducta prosocial para adultos. Revista Iberoamericana de Diagnóstico y Evaluación - e Avaliação Psicológica, 42(2), 15-25. doi:10.21865/ridep42_15

Balabanian, C., \& Lemos, V. (2016). Conducta prosocial adolescente: una propuesta para su operacionalización. Póster presentado en el VI Congreso Regional de la Sociedad Interamericana de Psicología. 15 al 17 de junio, Rosario, Argentina.

Bandura, A., Barbaranelli, C., Caprara, G. V., \& Pastorelli, C. (1996). Mechanisms of moral disengagement in the exercise of moral agency.Journal of Personality and Social Psychology, 71, 364-374.ISSN: 0022-3514

Caprara, G. V., Luengo Kanacri, B. P., Zuffiano, A., Gerbino, M., \& Pastorelli, C. (2015). Why and how to promote adolescents' prosocial behaviors: Direct, mediated and moderated effects of the CEPIDEA School-Based Program. Journal of Youth and Adolescence, 44(12), 2211-2229. doi:10.1007/s10964-015-0293-1.

Caprara, G. V., Steca, P., Zelli, A., \& Capanna, C. (2005).A new scale for measuring adults' prosocialness. European Journal of Psychological Assessment, 21(2), 77-89. doi: 10.1027/1015-5759.21.2.77

Carlo, G. \& Randall, B. (2002).The development of a measure of prosocial behaviors for late adolescents. Journal of Youth and Adolescence, 31(1), 31-44. doi 10.1023/a:1014033032440

Carlo, G., Fabes, R. A., Laible, D., \& Kupanoff, K. (1999). Early adolescence and prosocial/moral behavior II: The role of social and contextual influences. The Journal of Early Adolescence, 19(2), 133-147. doi:https://doi.org/10.1177/0272431699019002001

Cohen, R. J. \& Swerdlik, M. E. (2001). Pruebas y evaluación psicológicas. Introducción a las pruebas y a la medición.México: McGrawHill.

Davis, M. H. (1980). A multidimensional approach to individual differences in empathy. JSAS Catalog of Selected Documents in Psychology, 10, 85-104.
Del Barrio, V., Moreno Rosset, C., \& López Martínez, R. (2001). Evaluación de la agresión y la inestabilidad emocional en niños españoles: $\mathrm{Su}$ relación con la depresión. Clínica y Salud, 12(1), 33-50. ISSN: 1130-5274

Eisenberg, N., Fabes, R.A., \& Spinrad, T. L. (2006). "Prosocial development". In N. Eisenberg, W. Damon, \& R. M. Lerner. Handbook of Child Psychology. Vol. 3: Social, Emotional, and Personality Development, 646-718. 6th ed. Hoboken, NJ, EUA: John Wiley \& Sons.

Garaigordobil, M. (2005). Diseño y evaluación de un programa de intervención socioemocional para promover la conducta prosocial y prevenir la violencia. Madrid: Centro de Publicaciones del Ministerio de Educación y Ciencia.

Grant, A., \& Dutton, J. (2012). Beneficiary or benefactor: Are people more prosocial when they reflect on receiving or giving? Psychological Science, 23(9), 1033-1039. doi: 10.1177/0956797612439424

Greenberg, M. T., Weissberg, R. P., O’Brien, M. U., Zins, J. E., Fredericks, L., Resnik, H., \& Elias, M. J. (2003). Enhancing school-based prevention and youth development through coordinated social, emotional, and academic learning. American Psychologist, 58, 466474.ISSN: 0003-066X

Inglés, C. J., Benavides, G., Redondo, J., GarcíaFernández, J. M., Ruiz-Esteban, C., Estévez, C., \& Huescar, E. (2009). Conducta prosocial y rendimiento académico en estudiantes españoles de Educación Secundaria Obligatoria. Anales de Psicología, 25(1), 93101. ISSN: $1695-2294$

Jennings, P. A., \& Greenberg, M. T. (2009). The prosocial classroom: Teacher social and emotional competence in relation to child and classroom outcomes. Review of Educational Research, 79, 491-525. doi: 10.3102/00346543083256

Lemos, V. (2009). Evaluación de la eficacia de un programa para promover la conducta prosocial en niños en riesgo social por pobreza. En M. C. Richaud de Minzi y J. E. Moreno (Eds.). Recientes Desarrollos Iberoamericanos en Investigación en Ciencias 
del Comportamiento. Vol 1, pp. 137-152. ISBN 987-950-692-091-3. Buenos Aires: Ediciones CIIPME-CONICET.

Lemos, V. (2012). Estilos parentales, conducta prosocial y estabilidad emocional en niños en vulnerabilidad social por pobreza. En Palomar Lever, J. (Comp.). Estudios de Resiliencia en América Latina. México: PearsonUniversidad Iberoamericana-Universidad de Sonora. Pp. 11-22.

Lemos, V., Hendrie, K., \& Oros, L. (2015). Simpatía y su incidencia en la conducta prosocial en niños de 6 y 7 años. Revista de Psicología, 11(21), 47-59. ISSN: 1669-2438.

Lemos, V., \& Richaud de Minzi, M. C. (2013). Childhood prosocial behavior in the school environment. En A. Castro Solano (Ed.), Positive Psychology in Latin America. Netherlands: Springer.

Lemos, V., \& Richaud de Minzi, M. C. (2014). Promotion of child prosocial behavior in the school context. En A. Castro Solano (Ed.), Positive Psychology in Latin America. Editorial Springer.

López, F., \& Ortiz, M. J. (2005). El desarrollo del apego durante la infancia. En F. López, I. Etxebarría, M.J. Fuentes \& M.J. Ortiz (coords), Desarrollo afectivo y social, 41-66. Madrid, España: Pirámide.

López, M., Arán, V., \& Richaud de Minzi, M.C. (2014). Empatía: Algunos debates en torno al concepto. Avances de Psicología Latinoamericana 32, 37-51.

Martínez González, A. E., Inglés Saura, C., Piqueras Rodríguez, J. A., \& Oblitas Guadalupe, L. A. (2010). Papel de la conducta prosocial y de las relaciones sociales en el bienestar físico y psíquico del adolescente. Avances en Psicología Latinoamericana, 28(1), 74-84. ISSN:1794-4724

Martínez-Arias, M. R., Hernández-Lloreda, M. J., \& Hernández-Lloreda, M. V. (2006). Psicometría. Madrid: Alianza Editorial.

Martorell, C., González, R., Ordoñez, A., \& Gómez, O. (2011). Estudio confirmatorio del Cuestionario de Conducta Prosocial (CCP) y su relación con variables de personalidad y socialización. Revista Iberoamericana de Diagnóstico y Evaluación - e Avaliação Psicológica, 32(2), 35-52. ISSN: 1135-3848
Mestre Escrivá, M. V., Samper, P., Tur, A. M., Cortés, M. T., \& Nácher, M. J. (2006). Conducta prosocial y procesos psicológicos implicados: Un estudio longitudinal. Revista Mexicana de Psicología, 23(2), 203215.ISSN: 0185-6073

Montero, I., \& León, G. (2007). A guide for naming research studies in Psychology. International Journal of Clinical and Health Psychology, 7(3), 847-862.ISSN 1697-2600

Redondo Pacheco, J., Rueda Rueda, S., \& Amado Vega, C. (2013).Conducta prosocial: Una alternativa a las conductas agresivas. Investigium Ire: Ciencias Sociales $y$ Humanas, 4(1), 234-247.ISSN: 2216 - 1473

Richaud de Minzi, M. C. (2008). Estudio del IRI de Davis en población infantil argentina. Revista de Investigación en Psicología. Universidad de San Marcos, 11(1), 101-115.

Richaud de Minzi, M. C. (2014). Algunos aportes sobre la importancia de la empatía y la prosocialidad en el desarrollo humano. Revista Mexicana de Investigación en Psicología, 6(2), 171-176. ISSN: 2007-3240

Richaud de Minzi, M. C. (2015). Informe final proyecto de investigación subsidiado por CONICET. Influencia de la Empatía y de los procesos cognitivo-emocionales y sociales relacionados, sobre las conductas prosociales y agresivas. PIP No 11220100100230.

Richaud de Minzi, M. C., Lemos, V., \& Mesurado, B. (2011). Relaciones entre la percepción que tienen los niños de los estilos de relación y de la empatía de los padres y la conducta prosocial en la niñez media y tardía. Avances en Psicología Latinoamericana, 29(2),330-343. ISSN:1794-4724

Richaud de Minzi, M. C., Mesurado, B., \& Kohan Cortada, A. (2012). Analysis of dimensions of prosocial behavior in an Argentinean simple of children. Psychological Reports: Mental \& Physical Health 111(3), 687-696. doi:https://doi.org/10.2466/10.11.17.pr0.111.6.687-696

Roche Olivar, R. (1995). Psicología y educación para la prosocialidad. Buenos Ares: Ciudad Nueva.

Roche Olivar, R. (1997). La educación a la prosocialidad como optimizadora de la salud mental y de la calidad en las relaciones sociales. Trabajo presentado en el II 
Seminario de Psicología de la Educación: Comunicación y Salud Mental. Organizado por el Departament de Psicologia de 1 'Educació. Universitat Autònoma de Barcelona.

Roche Olivar, R. (1998). El uso educativo de la televisión como optimizadora de la prosocialidad. Psychosocial Intervention, 7(3), 363-378.

Samper García, P. (2014). Diferentes tendencias prosociales: El papel de las emociones. Revista mexicana de Investigación en Psicología, 6(2), 177-185. ISSN: 2007-3240

Seligman, M., \& Csikszentmihalyi, M. (2000). Positive psychology: An introduction. American Psychologist, 55, 5-14.doi: 10.1037//0003-066x.55.1.5

Tornimbeni, S., Peréz, E., \& Olaz, F. (2008). Introducción a la psicometría. Buenos Aires: Paidós. 